\title{
Abnormal Sleep Duration as Predictor for Cardiovascular Diseases: A Systematic Review of Prospective Studies
}

\author{
Sidhi Laksono $\mathbb{D},{ }^{1}$ Mefri Yanni $\mathbb{D},{ }^{2}$ Mohammad Iqbal $\mathbb{D},{ }^{3}$ and Ananta Siddhi Prawara $\mathbb{D}^{4}$ \\ ${ }^{1}$ Department of Cardiology and Vascular Medicine, RS Pusat Pertamina, Faculty of Medicine, Universitas Muhammadiyah Prof. \\ DR. Hamka, Tangerang, Indonesia \\ ${ }^{2}$ Department of Cardiology and Vascular Medicine, RSUP M. Djamil, Faculty of Medicine, Universitas Andalas, Padang, Indonesia \\ ${ }^{3}$ Department of Cardiology and Vascular Medicine, RSUP Hasan Sadikin, Faculty of Medicine, Universitas Padjadjaran, \\ Bandung, Indonesia \\ ${ }^{4}$ Faculty of Medicine, Universitas Diponegoro, Semarang, Indonesia
}

Correspondence should be addressed to Sidhi Laksono; sidhilaksono@uhamka.ac.id

Received 12 March 2021; Accepted 20 January 2022; Published 7 February 2022

Academic Editor: Siraj Omar Wali

Copyright ( 2022 Sidhi Laksono et al. This is an open access article distributed under the Creative Commons Attribution License, which permits unrestricted use, distribution, and reproduction in any medium, provided the original work is properly cited.

Study Objective. This study is aimed at figuring out the role of abnormal sleep duration in predicting cardiovascular diseases. Methods. We searched the online database PubMed on 30 August 2020 for our data collection. We used the following keywords: sleep duration AND (cardiovascular disease OR cardiovascular event) AND (cohort OR prospective OR retrospective). We identified 653 studies, and after excluding studies that were published before 2015, we obtained 306 studies. After filtering the 306 studies through title and abstract screening and applying the inclusion and exclusion criteria, we further reviewed fourteen studies with full-text reading. We excluded three studies because of insufficient data required and included eleven studies in this systematic review. Results. A total of 361,041 participants from ten studies were included in this systematic review. The incidence of hypertension, myocardial infarction, coronary artery disease, heart failure, cardiovascular events, and cardiovascular diseases in the short sleep duration group is $46.12 \%, 0.59 \%, 5.43 \%, 0.09 \%, 7.18 \%, 1.48 \%$, and $6.8 \%$, consecutively, while the incidence of hypertension, myocardial infarction, coronary artery disease, and heart failure in the long sleep duration group is $30.71 \%, 0.61 \%, 6.55 \%, 1.11 \%$, and $6.04 \%$, consecutively. Nine studies reported an association between sleep duration and cardiovascular diseases while one study reported no association. Seven studies reported that short sleep duration was significantly associated with CVD. Short sleep duration in this study was associated with hypertension and heart failure. Atrial fibrillation and coronary artery disease were associated with both short and long sleep duration. Conclusion. Abnormal sleep duration (short and long sleep duration) may act as the predictor of cardiovascular diseases. The importance of having normal sleep duration should be stressed with other lifestyle modification to avoid the risk of getting cardiovascular diseases. However, further studies are needed to overcome the limitation of this systematic review.

\section{Introduction}

Cardiovascular diseases, a group of disorders of the heart and blood vessels, were responsible for the highest death rate in the world. It was estimated to be the cause of death of 17.9 million people in 2017 [1]. The advancement of cardiovascular diseases' management that can be seen in the recent guidelines published by the European Society of Cardiology (ESC), American College of Cardiology (ACC), and International Society of Hypertension (ISH) brings hope to reduce the morbidity and mortality of the patients with cardiovascular diseases. The importance of lifestyle modifications (including balanced diet, physical activity, and obstructive sleep apnea management) has been stressed on the published guidelines [2-10]. However, the significance of having enough sleep was not yet included in the guidelines, even though it might further help the patients, especially in preventing further cardiovascular diseases.

The prevalence of people with a sleep disorder or report insufficient sleep has increased; it was reported by the National 
Heart, Lung, and Blood Institute of the National Institutes of Health that approximately 50 to 70 million American adults suffer from sleep disorder [11]. The increasing prevalence of people with abnormal sleep duration may be caused by an increase in activities that operate 24/7 and usage of electronics such as mobile phones [12]. More activities that operate 24/7 resulted in more workers working under shift works. Different shift works (morning, afternoon, and night shifts) have different impacts to the quantity and quality of sleep as described by Åkerstedt [13], while the increasing use of electronics including mobile phones during the day and bedtime have a negative correlation with sleep duration [14]. The American Academy of Sleep Medicine (AASM) and Sleep Research Society (SRS) published a consensus in 2015 which defined normal sleep duration as sleep 7 or more hours per night. However, the consensus also stated that sleeping more than 9 hours per night was associated with health risk for those who were not included in one of these: young adults, individuals recovering from sleep debt, and individuals with illness [15]. Abnormal sleep duration, especially short sleep duration, was known to increase the sympathetic tone from the predominant neutral interaction of sympathovagal. These changes in autonomic dysregulation led to cardiovascular diseases [16]. In another study, it was also suggested that abnormal sleep duration may alter circadian clock genes which acted as a central regulator of the circadian rhythm [17].

Circadian rhythm in the human body is generated by two major clocks: the central clock and the peripheral clock. The central clock refers to the hypothalamic master clock in the suprachiasmatic nucleus (SCN). Changes of light, which was detected by the retinal cells of the eyes, signal the central clock to activate the transcription of Clock (circadian locomotor output cycles kaput) and Bmall (brain and muscle Arnt-like protein 1) genes. These genes played an important role in activating molecular sequences of the circadian rhythm [18]. On the other hand, the peripheral clock refers to the intrinsic circadian rhythms in each organ system, including the cardiovascular system, which may act independently from the central clock [19]. The circadian mechanism can be regulated in the absence of light/dark cycles by food intake, temperature, and environment. The environmental stimuli may affect circadian rhythm specifically according to the cells and tissues; they were known as zeitgebers. The zeitgebers also regulate circadian rhythm in cardiomyocytes, fibroblasts, and vascular smooth muscle cells [18]. Alterations of central and/or peripheral circadian rhythm because of abnormal sleep duration may cause abnormality in hormonal secretion, blood pressure, heart rate, endothelial cell function, platelet aggregation, thrombus formation, and other physiological processes $[18,20]$. Thus, we are aiming to figure out the role of abnormal sleep duration in predicting cardiovascular diseases. Our systematic review will provide a better evidence regarding abnormal sleep duration and cardiovascular outcomes. There are no systematic review regarding abnormal sleep duration and hypertension.

\section{Methods}

This review has been reported following the Preferred Reporting Items for Systematic Reviews (PRISMA) guide- lines 2015. We used population (patient with sleep disorder), intervention/exposure (sleep disorders), comparator (without sleep disorders), outcome (cardiovascular disease), and study design (cohorts) using the PICOs framework to formulate the research question.

2.1. Inclusion and Exclusion Criteria. We evaluated English language papers published within five years that evaluate the association between sleep duration and cardiovascular diseases. We included only prospective cohort studies that were previously peer reviewed and all participants that did not have prior specific cardiovascular diseases that were being measured by each study included. We excluded surveys, cross-sectional studies, case-control studies, and Mendelian randomization.

2.2. Types of Outcome Measurement. The measurement of sleep duration could be done objectively or subjectively. Sleep duration is divided as short and long sleep duration. Short sleep duration was defined as sleep less than or equal to 4.9 hours to less than 7 hours, while long sleep duration was defined as sleep more than or equal to 7.5 hours to more than or equal to 10 hours. Questionnaires or interview are considered as subjective measurement of sleep duration. The performance of subjective measurement of sleep duration was known to vary according to demographic and did not closely correspond to the objective measurement of sleep $[19,20]$. However, this method was more efficient and practicable in collecting sleep duration data in a large population study, which suited the condition of the 10 studies [21]. Sleep duration was assessed with questionnaires in 9 studies and interviews in 2 studies by asking how many hours they slept on average during the month in one study and how many hours they slept usually in a day in another. Short sleep duration and long sleep duration were defined differently in the studies. Short sleep duration was defined as sleep less than or equal to 4.9 hours to less than 7 hours, while long sleep duration was defined as sleep more than or equal to 7.5 hours to more than or equal to 10 hours.

The primary outcome included in this study is the duration of sleep and cardiovascular disease incident in patients that have no prior specific cardiovascular diseases that was evaluated by each study. The secondary outcomes included in this study are the number of participants, follow-up duration (assessed from follow-up information provided in the study), participants' age (assessed from reported age information in the study), male percentage (assessed from reported numbers of male in the study divided by the total participants), and sleep assessment tool used (assessed from information regarding tools being used in the study).

2.3. Electronic Search. Two independent authors searched the online database PubMed on 30 August 2020 for our data collection. We used the following keywords: sleep duration AND (cardiovascular disease OR cardiovascular event) AND (cohort OR prospective OR retrospective).

2.4. Study Selection. 653 studies were identified, and after excluding studies that were published before 2015 (latest study, study from the last 5 years), 306 studies were 
obtained. After filtering the 306 studies through title and abstract screening and applying the inclusion and exclusion criteria, thirteen studies were further reviewed with full-text reading. Three studies were excluded because of insufficient data required. Thus, ten studies were included in this systematic review. The study consort diagram can be seen in Figure 1. Two authors (SL and ASP) independently evaluated the title and abstracts of the retrieved articles. The selected articles in the first step of evaluation had their full text assessed by the same reviewers. In cases of any disagreements in the previous steps, decision will be taken after discussion. We used the Newcastle-Ottawa quality assessment scale (NOS) for cohort studies to evaluate the quality of included studies. In the current study, we considered a study awarded six or more points as a high quality study. Discrepancies were resolved by discussion. In this study, 3 studies are classified as high risk and 7 studies as high-quality studies. Follow-up varies from 3 to 20 years.

2.5. Data Extraction. Two authors (SL and ASP) also performed data extraction and study quality assessment independently. Data extraction was organized in spreadsheets. The extracted data of each study included the following information: the authors' name, year of publication, country, number of participants, baseline year, follow-up duration, mean participants' age, male percentage, sleep assessment tool, outcome assessed, sleep category, normal sleep duration, the result (incidence, OR/HR), conclusion, and adjusted variables. The meta-analysis was not feasible due to different definitions of sleep disorder; this different definition caused a different exposure too.

\section{Results}

A total of 361,041 participants from 10 prospective cohort studies published between 2015 and 2020 were included in this systematic review. The studies included were conducted in 6 countries and 3 continents. The cardiovascular diseases assessed by the 10 studies differed; 3 assessed hypertension, 1 assessed myocardial infarction, 2 assessed coronary heart disease, 1 assessed atrial fibrillation, 1 assessed heart failure, 1 assessed cardiovascular disease (myocardial infarction, angina pectoris, revascularization procedure, and stroke), and 1 assessed cardiovascular events (atrial fibrillation, myocardial infarction, and stroke). The baseline information of the studies can be seen in Table 1 .

The 9 studies included in this systematic review reported an association between sleep duration and cardiovascular diseases while 2 studies reported no association. From the 9 studies which reported the association, 7 reported that short sleep duration was significantly associated with CVD. Short sleep duration in this study was associated with hypertension, coronary heart disease, and cardiovascular events (atrial fibrillation, myocardial infarction, and stroke). Odds ratio from study being used in this review varies from 0.89 to 3.15. Most of the studies could not control all of confounders, while some control few of its confounders. On the other hand, 2 studies reported that long sleep duration was significantly associated with CVD. The CVDs that were associated with long sleep duration were atrial fibrillation and coronary heart disease (nonfatal MI, stable angina, unstable angina, unspecified CHD, or CHD death). Hypertension was not cited as exclusion criteria in 8 studies which are aimed at finding an association between sleep duration and the occurrence of other cardiovascular diseases. However, we still included the 8 studies because blood pressure or hypertension was still included as one of the adjusted variables when the studies find the association between sleep duration and cardiovascular disease. The association between sleep duration and cardiovascular disease from 10 studies can be seen in Table 2 .

\section{Discussion}

Overall, the evidence might suggest association between abnormal sleep duration and cardiovascular outcome. Three studies show short sleep duration was consistently associated with the occurrence of hypertension in middle-aged participants (both men and women) [22-24]. Four included studies reported that two cardiovascular diseases, coronary heart diseases and atrial fibrillation, are associated with both long and short sleep duration. Two studies in this systematic review assessed a more general outcome which was cardiovascular events and cardiovascular diseases [25-29].

4.1. Abnormal Sleep Duration and Cardiovascular Diseases. Short sleep duration was consistently associated with the occurrence of hypertension in middle-aged participants (both men and women) from the three studies [22-24]. The study conducted by Mallinson et al. specifically mentioned that the occurrence of hypertension in women was higher [23]. This finding was supported by a previous study conducted by Bertisch et al. which also stated that short sleep duration in women was associated with hypertension compared to women who have normal sleep duration [30]. Align with hypertension, coronary artery calcification, and heart failure were associated with short sleep duration [28, 31]. Multiple mechanisms, not one, are responsible for the development of cardiovascular diseases from short sleep duration. The mechanisms are the increase of proinflammatory cytokines, sympathetic activity, oxidative stress, hypercholesterolemia, ghrelin, and the decrease of leptin. These mechanisms may be linked to circadian misalignment directly or indirectly [32].

Four studies have reported that two cardiovascular diseases, coronary heart diseases and atrial fibrillation, were found to be associated with both long and short sleep duration [25-28]. Tobaldini et al. found that fibrinogen may be responsible as the mechanism underlying the coronary heart disease occurrence in participants with long sleep duration even though the exact mechanism is not understood yet [33]. A systematic review that was aimed at figuring out the association of atrial fibrillation and sleep duration conducted by Morovatdar et al. found a similar result with the findings in this systematic review in which atrial fibrillation was associated with short and long sleep duration. However, the authors mentioned that the exact mechanism between atrial fibrillation and sleep duration remains unclear [34]. 


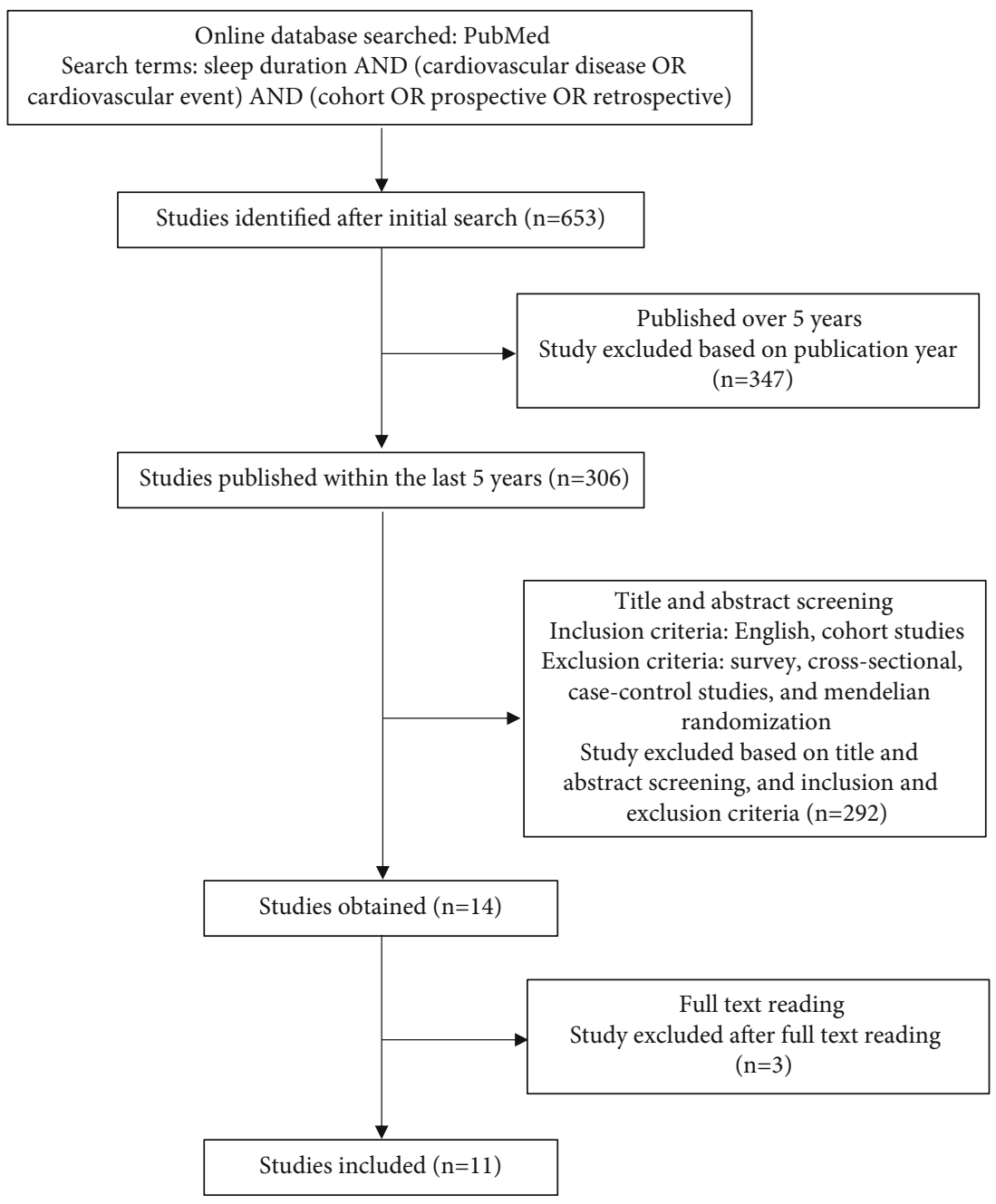

Figure 1: Study consort diagram of the systematic review.

In a different study, it was found that sleep deprivation caused a reduction in left atrium early diastolic strain rate, which in the long term may develop into atrial fibrillation [35]. Generally, long sleep duration can be associated with an increased risk of CVD because long sleep duration itself was associated with depressive symptoms, low socioeconomic status, unemployment, and low physical activity. These conditions that were associated with long sleep duration can also act as CVD risk factors [36].

Myocardial infarction was not reported to be associated with abnormal sleep duration in a study conducted by Song et al. [37]. The association was also not found when myocardial infarction was being assessed as a component of cardiovascular diseases, except when the participants have short sleep duration and insomnia [29]. The association of myocardial infarction and sleep duration was found when myocardial infarction was assessed as a component of either coronary heart disease [25] (long sleep duration) or cardiovascular events [28] (low stable pattern).

Two studies in this systematic review assessed a more general outcome which was cardiovascular events and cardiovascular diseases. Cardiovascular events were stated to be associated with low stable sleep pattern, followed by low increasing sleep pattern. These findings suggest that despite the follow-up sleep duration, the short sleep duration in the baseline can predict cardiovascular events. However, the finding can also be interpreted that the risk of the occurrence of cardiovascular events can be reduced by increasing sleep duration [28]. The association of short sleep duration and cardiovascular diseases was not found in the study conducted by Wang et al., but the association was found when assessing the relation between patients with short sleep duration and insomnia/poor sleep with cardiovascular diseases [29]. This finding suggested that besides sleep duration, altered sleep quality (insomnia/poor sleep) may also affect cardiovascular diseases.

The findings from the studies included in this systematic review suggest that both short sleep duration and long sleep duration may be associated with cardiovascular diseases. A previous study conducted by Kwok et al. has confirmed that there was a U-shaped relation between sleep duration and hypertension [38]. Guo et al. also found a U-shaped relation for $\mathrm{CHF}$, stroke, and coronary artery disease with sleep duration in their study [39]. 


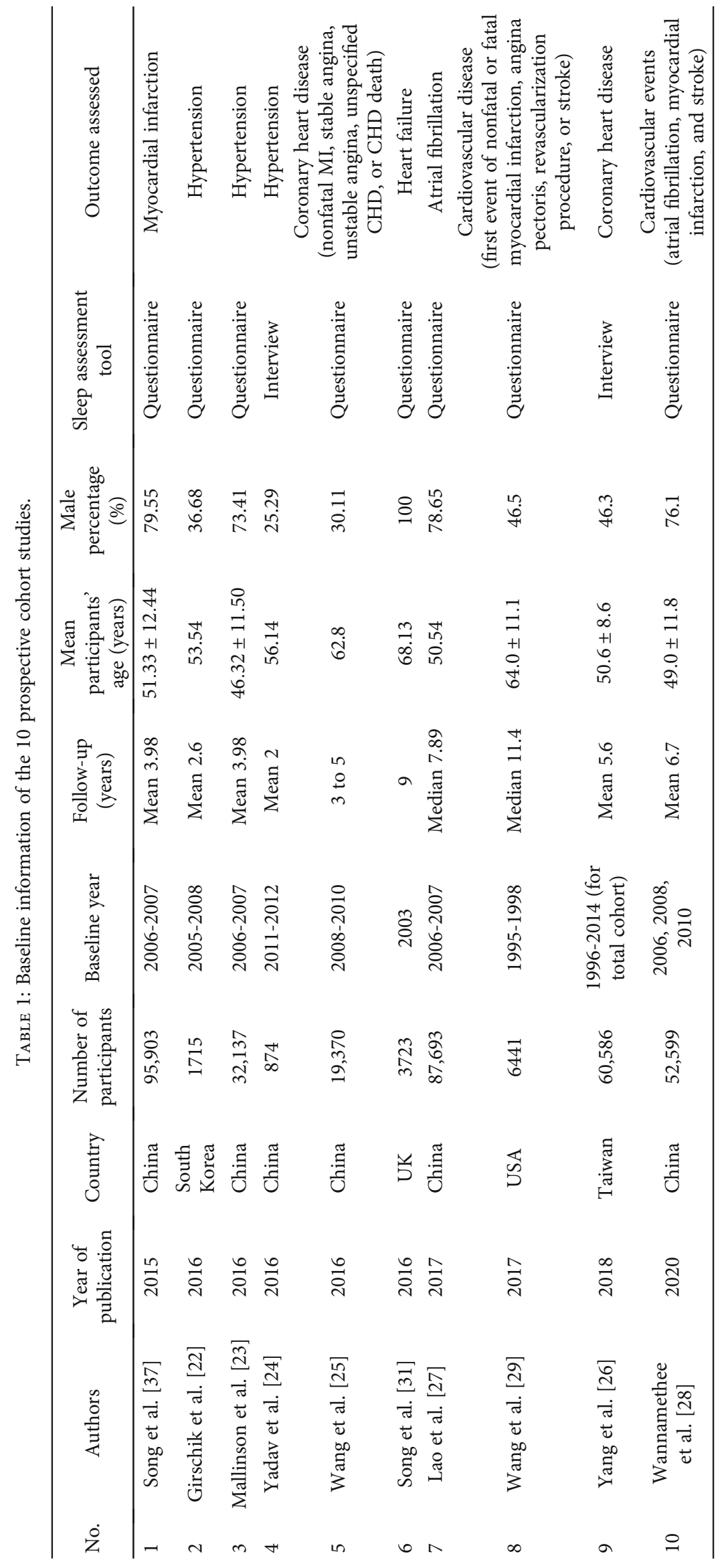




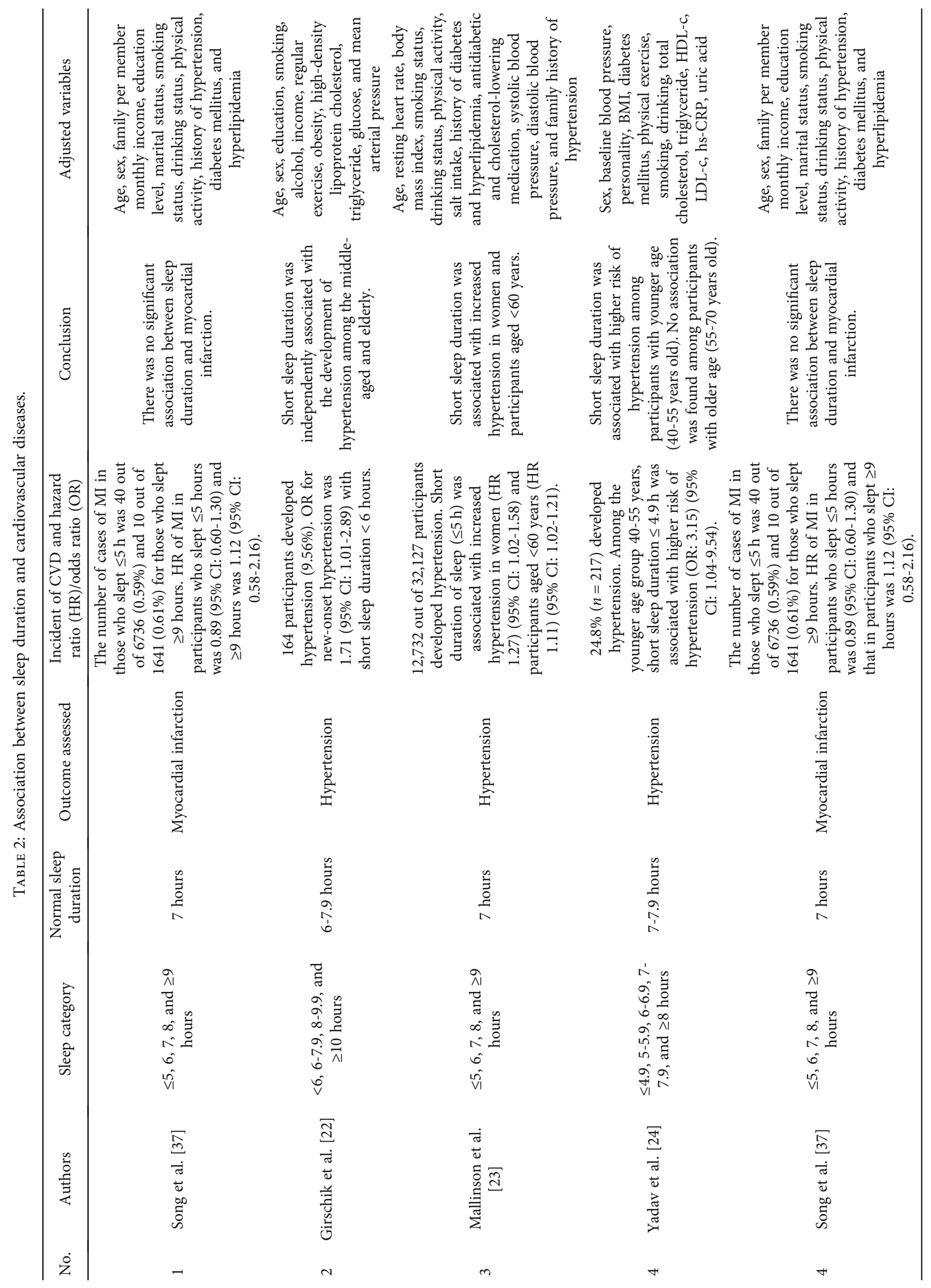




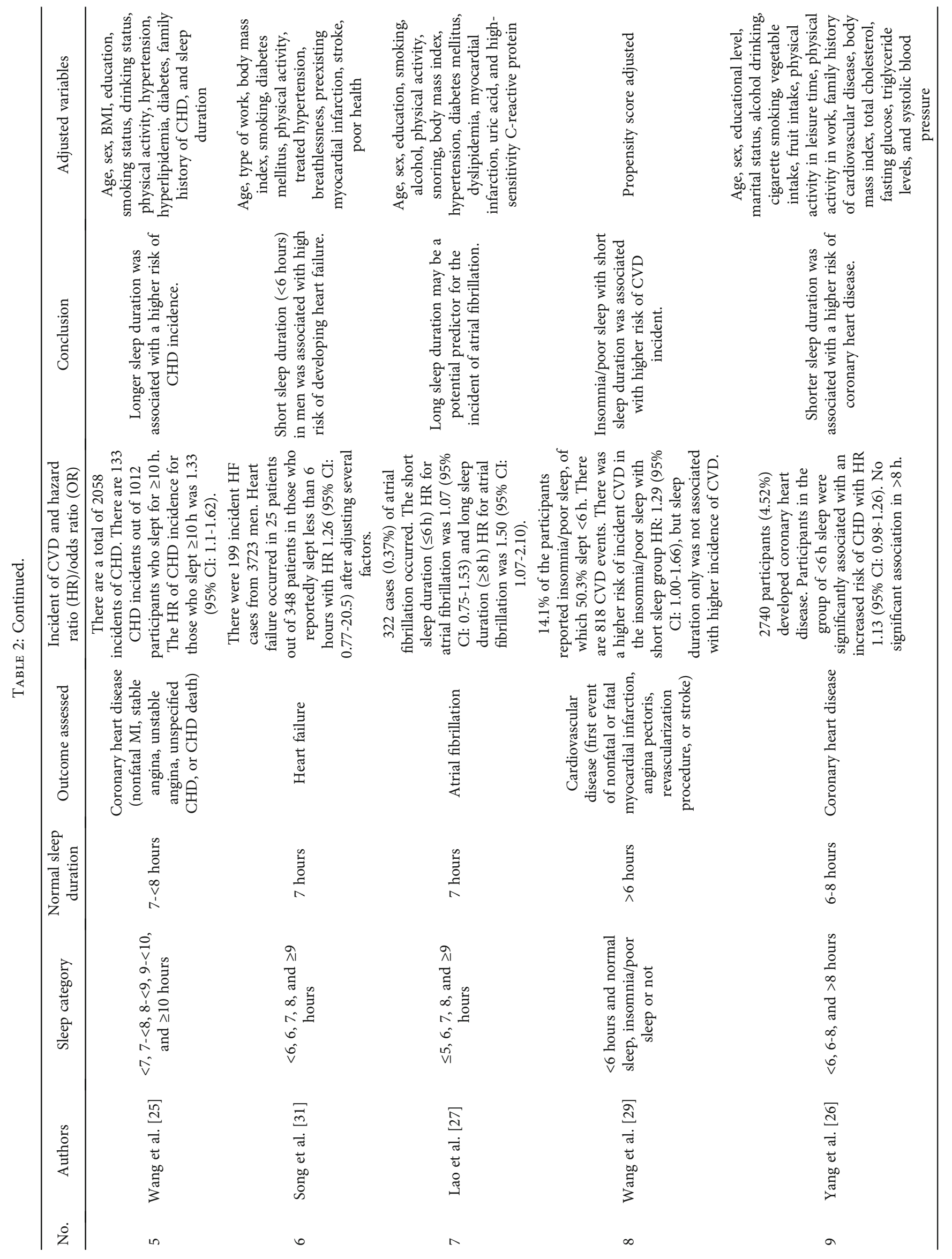




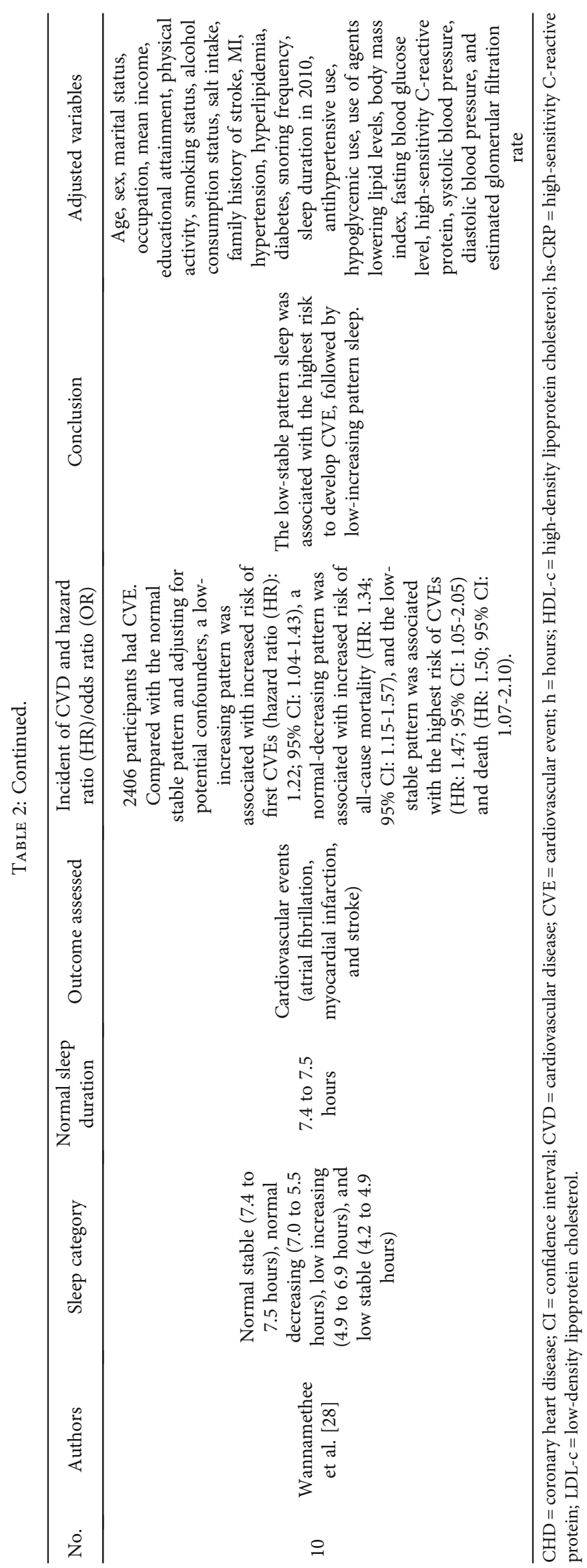


4.2. Normal Sleep Duration. The 10 studies defined normal sleep duration differently. Four studies defined 7 hours as normal sleep duration. Seven other studies defined normal sleep duration using range: $>6$ hours, 6-7.9 hours, 6-8 hours, 7-7.9 hours, 7-<7.5 hours, 7-<8 hours, and 7.4-7.5 hours. The differences of normal sleep duration of the 10 studies may be due to the baseline year of when the studies began before the publication of the consensus.

4.3. Study Limitations. There are several limitations to this systematic review. First, normal sleep duration was defined differently in the studies included in this study. The first limitation limited us on performing a meta-analysis. Second, sleep duration in the studies included in this systematic review was measured subjectively. Objective measurements can be considered in future studies. Third, we did not assess the occurrence of obstructive sleep apnea in this systematic review. Fourth, besides sleep duration, sleep patterns and sleep quality can also affect the cardiovascular system. Thus, a further study explaining sleep patterns and sleep quality is needed to complete the information about sleep that can be given to the patients.

\section{Conclusion}

Both short and long sleep duration was associated with cardiovascular diseases compared to normal sleep duration. Short sleep duration was associated with hypertension, coronary artery calcification, and heart failure. Coronary heart disease and atrial fibrillation can be associated with both short and long sleep duration. The importance of having normal sleep duration should be stressed during educating the patients, besides other lifestyle modifications. Further studies are needed to overcome the limitation of this systematic review.

\section{Conflicts of Interest}

There is none related to this manuscript.

\section{Acknowledgments}

The authors thank all who support this systematic review.

\section{References}

[1] G. A. Mensah, G. A. Roth, and V. Fuster, "The global burden of cardiovascular diseases and risk factors: 2020 and beyond," Journal of the American College of Cardiology, vol. 74, no. 20, pp. 2529-2532, 2019.

[2] Bryan Williams, Giuseppe Mancia, Wilko Spiering et al., "2018 ESC/ESH Guidelines for the management of arterial hypertension. The Task Force for the management of arterial hypertension of the European Society of Cardiology (ESC) and the European Society of Hypertension (ESH)," Giornale Italiano di Cardiologia, vol. 11, Supplement 1, pp. 3S-73S, 2018.

[3] P. K. Whelton, R. M. Carey, W. S. Aronow et al., "2017 ACC/ AHA/AAPA/ABC/ACPM/AGS/APhA/ASH/ASPC/NMA/ PCNA Guideline for the Prevention, Detection, Evaluation, and Management of High Blood Pressure in Adults: Executive Summary: A Report of the American College of Cardiology/
American Heart Association Task Force on Clinical Practice Guidelines," Hypertension, vol. 71, no. 6, pp. 1269-1324, 2018.

[4] T. Unger, C. Borghi, F. Charchar et al., "2020 International Society of Hypertension global hypertension practice guidelines," Hypertension, vol. 75, no. 6, pp. 1334-1357, 2020.

[5] G. Hindricks, T. Potpara, N. Dagres et al., "2020 ESC guidelines for the diagnosis and management of atrial fibrillation developed in collaboration with the European Association of Cardio-Thoracic Surgery (EACTS)," European Heart Journal, vol. 42, no. 5, pp. 373-498, 2021.

[6] J.-P. Collet, H. Thiele, E. Barbato et al., "ESC guidelines for the management of acute coronary syndromes in patients presenting without persistent ST-segment elevation," European Heart Journal, vol. 2020, pp. 1-79, 2021.

[7] B. Ibanez, S. James, S. Agewall et al., "2017 ESC guidelines for the management of acute myocardial infarction in patients presenting with ST-segment elevation," European Heart Journal, vol. 39, no. 2, pp. 119-177, 2018.

[8] P. Ponikowski, A. A. Voors, S. D. Anker et al., "2016 ESC guidelines for the diagnosis and treatment of acute and chronic heart failure," European Heart Journal, vol. 37, no. 27, pp. 2129-2200, 2016.

[9] C. T. January, L. S. Wann, H. Calkins et al., "2019 AHA/ACC/ HRS focused update of the 2014 AHA/ACC/HRS guideline for the management of patients with atrial fibrillation: a report of the American College of Cardiology/American Heart Association Task Force on Clinical Practice Guidelines and the Heart Rhythm Society," Journal of the American College of Cardiology, vol. 74, no. 1, pp. 104-132, 2019.

[10] C. W. Yancy, M. Jessup, B. Bozkurt et al., "2017 ACC/AHA/ HFSA focused update of the 2013 ACCF/AHA guideline for the management of heart failure: a report of the American College of Cardiology/American Heart Association Task Force on Clinical Practice Guidelines and the Heart Failure Society of America," Journal of the American College of Cardiology, vol. 70, no. 6, pp. 776-803, 2017.

[11] M. P. St-Onge, M. A. Grandner, D. Brown et al., "Sleep duration and quality: impact on lifestyle behaviors and cardiometabolic health: a scientific statement from the American Heart Association," Circulation, vol. 134, no. 18, pp. e367-e386, 2016.

[12] Y. Serin and N. A. Tek, "Effect of circadian rhythm on metabolic processes and the regulation of energy balance," Annals of Nutrition \& Metabolism, vol. 74, no. 4, pp. 322-330, 2019.

[13] T. Åkerstedt, "Shift work and disturbed sleep/wakefulness," Sleep Medicine Reviews, vol. 2, no. 2, pp. 117-128, 1998.

[14] M. Hysing, S. Pallesen, K. M. Stormark, R. Jakobsen, A. J. Lundervold, and B. Sivertsen, "Sleep and use of electronic devices in adolescence: results from a large population-based study," BMJ Open, vol. 5, no. 1, article e006748, 2015.

[15] Consensus Conference Panel, N. F. Watson, M. S. Badr et al., "Recommended amount of sleep for a healthy adult: a joint consensus statement of the American Academy of Sleep Medicine and Sleep Research Society," Journal of Clinical Sleep Medicine, vol. 11, no. 6, pp. 591-592, 2015.

[16] M. Nagai, S. Hoshide, and K. Kario, "Sleep duration as a risk factor for cardiovascular disease- a review of the recent literature," Current Cardiology Reviews, vol. 6, no. 1, pp. 54-61, 2010.

[17] H. Liu and A. Chen, "Roles of sleep deprivation in cardiovascular dysfunctions," Life Sciences, vol. 219, no. 253, pp. 231237, 2019. 
[18] I. Rabinovich-Nikitin, B. Lieberman, T. A. Martino, and L. A. Kirshenbaum, "Circadian-regulated cell death in cardiovascular diseases," Circulation, vol. 139, no. 7, pp. 965980, 2019.

[19] C. S. Mcalpine and F. K. Swirski, "Circadian influence on metabolism and inflammation in atherosclerosis," Circulation Research, vol. 119, no. 1, pp. 131-141, 2016.

[20] I. M. Hower, S. A. Harper, and T. W. Buford, "Circadian rhythms, exercise, and cardiovascular health," Journal of Circadian Rhythms, vol. 16, no. 1, pp. 1-8, 2018.

[21] E. M. Cespedes, F. B. Hu, S. Redline et al., "Comparison of selfreported sleep duration with actigraphy: results from the Hispanic community health study/study of Latinos Sueño Ancillary Study," American Journal of Epidemiology, vol. 183, no. 6, pp. 561-573, 2016.

[22] J. Girschik, L. Fritschi, J. Heyworth, and F. Waters, "Validation of self-reported sleep against actigraphy," Journal of Epidemiology, vol. 22, no. 5, pp. 462-468, 2012.

[23] D. C. Mallinson, M. E. Kamenetsky, E. W. Hagen, and P. E. Peppard, "Subjective sleep measurement: comparing sleep diary to questionnaire," Nature and Science of Sleep, vol. Volume 11, pp. 197-206, 2019.

[24] D. Yadav, D. S. Hyun, S. V. Ahn, S. B. Koh, J. Y. Kim, and Y. Kim, "A prospective study of the association between total sleep duration and incident hypertension," Journal of Clinical Hypertension, vol. 19, no. 5, pp. 550-557, 2017.

[25] X. Wang, X. Liu, Q. Song, and S. Wu, "Sleep duration and risk of myocardial infarction and all-cause death in a Chinese population: the Kailuan study," Sleep Medicine, vol. 19, pp. 13-16, 2016.

[26] L. Yang, H. Yang, M. He et al., "Longer sleep duration and midday napping are associated with a higher risk of CHD incidence in middle-aged and older Chinese : the DongfengTongji cohort study," Sleep, vol. 39, no. 3, pp. 645-652, 2016.

[27] X. Q. Lao, X. Liu, H. Deng et al., "Sleep quality, sleep duration, and the risk of coronary heart disease : a prospective cohort study with 60, 586 adults," Journal of Clinical Sleep Medicine, vol. 14, no. 1, pp. 109-117, 2018.

[28] S. G. Wannamethee, O. Papacosta, L. Lennon, and P. H. Whincup, "Self-reported sleep duration, napping, and incident heart failure: prospective associations in the British Regional Heart Study," Journal of the American Geriatrics Society, vol. 64, no. 9, pp. 1845-1850, 2016.

[29] Y. Wang, J. Wang, S. Chen et al., “Association of longitudinal patterns of habitual sleep duration with risk of cardiovascular events and all-cause mortality," JAMA Network Open, vol. 3, no. 5, pp. 1-15, 2020.

[30] S. M. Bertisch, B. D. Pollock, M. A. Mittleman et al., "Insomnia with objective short sleep duration and risk of incident cardiovascular disease and all-cause mortality : Sleep Heart Health Study," Sleep, vol. 41, no. 6, pp. 1-9, 2018.

[31] Q. Song, X. Liu, W. Hu et al., "Long sleep duration is an independent risk factor for incident atrial fibrillation in a Chinese population: a prospective cohort study," Scientific Reports, vol. 7, no. 1, pp. 1-7, 2017.

[32] J. E. Gangwisch, D. Feskanich, D. Malaspina, S. Shen, and J. P. Forman, "Sleep duration and risk for hypertension in women : results from the Nurses' Health Study," American Journal of Hypertension, vol. 26, no. 7, pp. 903-911, 2013.

[33] E. Tobaldini, E. M. Fiorelli, M. Solbiati, G. Costantino, L. Nobili, and N. Montano, "Short sleep duration and cardio- metabolic risk: from pathophysiology to clinical evidence," Nature Reviews. Cardiology, vol. 16, no. 4, pp. 213-224, 2019.

[34] L. Hale, V. Parente, J. B. Dowd et al., "Fibrinogen may mediate the association between long sleep duration and coronary heart disease," Journal of Sleep Research, vol. 22, no. 3, pp. 305-314, 2013.

[35] N. Morovatdar, N. Ebrahimi, R. Rezaee, H. Poorzand, M. A. B. Tork, and A. Sahebkar, "Sleep duration and risk of atrial fibrillation: a systematic review," Journal of atrial Fibrillation, vol. 11, no. 6, pp. 5-10, 2019.

[36] G. Açar, M. Akçakoyun, I. Sari et al., "Acute sleep deprivation in healthy adults is associated with a reduction in left atrial early diastolic strain rate," Sleep \& Breathing, vol. 17, no. 3, pp. 975-983, 2013.

[37] Q. Song, X. Liu, X. Wang, and S. Wu, "Age- and genderspecific associations between sleep duration and incident hypertension in a Chinese population: the Kailuan study," Journal of Human Hypertension, vol. 30, no. 8, pp. 503-507, 2016.

[38] C. S. Kwok, E. Kontopantelis, G. Kuligowski et al., "Selfreported sleep duration and quality and cardiovascular disease and mortality: a dose-response meta-analysis," Journal of the American Heart Association, vol. 7, no. 15, pp. 1-26, 2018.

[39] X. Guo, L. Zheng, J. Wang et al., "Epidemiological evidence for the link between sleep duration and high blood pressure: a systematic review and meta-analysis," Sleep Medicine, vol. 14, no. 4, pp. 324-332, 2013. 